\title{
An Efficient T-S Assimilation Strategy Based on the Developed Argo-Extending Algorithm
}

\author{
Chaojie Zhou,, ${ }^{1,2}$ Xiaohua Ding, ${ }^{1}$ Jie Zhang, ${ }^{2}$ Jungang Yang, ${ }^{2}$ and Qiang $\mathrm{Ma}^{1}$ \\ ${ }^{1}$ Department of Mathematics, Harbin Institute of Technology at Weihai, Weihai 264209, China \\ ${ }^{2}$ The First Institute of Oceanography, State Oceanic Administration, Qingdao 266061, China \\ Correspondence should be addressed to Chaojie Zhou; hitzcj@163.com
}

Received 22 June 2017; Accepted 11 September 2017; Published 16 October 2017

Academic Editor: Shaoqing Zhang

Copyright (c) 2017 Chaojie Zhou et al. This is an open access article distributed under the Creative Commons Attribution License, which permits unrestricted use, distribution, and reproduction in any medium, provided the original work is properly cited.

\begin{abstract}
Data assimilation is an efficient technique in the estimation of ocean state, by introducing the benefit of in situ measurements. Considering the insufficiency of the observations, the performance of assimilation with few temperature and salinity (T-S) profiles is not satisfied. To modify the situation, an extending algorithm based on the Argo temperature profile is developed and applied to present more reconstructed information. Meanwhile, when the reconstructed information is assimilated into the ocean model, the accuracy of the outcomes would obtain a notable enhancement. To validate it, an experiment including four cases is conducted based on Regional Ocean Modeling System (ROMS) and 4-dimensional variational method (4DVAR). The comparison with the EN4 dataset shows that the cases assimilated the Argo and the reconstructed temperature profiles are both promoted; the addition of reconstructed temperature profiles does enhance the accuracy; the impact of SST introduced in the extending algorithm process is negligible; the net enhancement of reconstructed temperature profiles is comparable with Argo T-S observations. Finally, the positive impact of the developed algorithm on data assimilation is validated.
\end{abstract}

\section{Introduction}

An accurate simulation of ocean state is essential to many oceanographic applications, such as the reanalysis of oceanic three-dimensional movements and the prediction of the climate variations [1-7]. Data assimilation is an efficient technique which could enhance the outcomes of model by taking advantage of consistency constraints with laws of time evolution and physical properties. Sufficient and accurate observations are demanded for a successful assimilation. Fortunately, global oceanic surface information could be provided by remote sensing, but its impact on the deep ocean is limited when it is assimilated directly. The combination with T-S profiles would modify the situation. Considering the limited observations in the subsurface, the research of efficient assimilation technology is becoming a significant issue.

The ocean surface states such as sea surface height (SSH) and sea surface temperature (SST) are the presentation of the complex dynamic behaviors in ocean and atmosphere.
Several methods have been developed to translate the surface observations to the subsurface, which can also be applied to benefit the assimilation. By taking the characteristic of water movement and energy exchange into consideration, Hurlburt [8] built a numerical ocean model to dynamically transfer simulated altimeter data into subsurface information. The model was able to reconstruct the deep pressure field even in situations with energetic shallow and deep circulations, baroclinic instability, and a vigorous vertical exchange of energy. Cooper and Haines [9] proposed a new method to assimilate the sea surface pressure by displacing the water columns vertically. An identical twin experiment was performed for 1 year with complete surface pressure data assimilated every 9 days and the error analysis gave an acceptable result, but this method cannot be applied to free-surface model. To meet the US Navy's need to produce rapid estimates of present and near-term ocean conditions, Fox et al. [10] combined in situ measurements, remotely sensed temperatures, and heights to form a single integrated analysis of temperature and salinity on a regular grid. But the parameters in the algorithm relied 
on the statistical analysis of T-S profiles. Yan et al. [11] developed a data assimilation scheme based on three-dimensional variational analysis (3DVAR) to estimate T-S profiles from surface dynamic height information. In the scheme, both vertical correlations for temperature and salinity background errors and the nonlinear temperature and salinity (T-S) relation were taken into consideration. Ratheesh et al. [12] explored the usefulness of satellite-derived surface data for nowcasting of oceanic circulation features, including SST and sea level anomaly (SLA). Surface information was projected into the vertical using predetermined correlation functions in combination with optimal interpolation, but the correlation functions were obtained based on large numbers of profiles.

Fortunately, the Argo program has built a real-time global ocean observation system, and the profiling floats have sampled the upper $2000 \mathrm{~m}$ of the ocean and provided freely available T-S observations with global coverage [13]. The assimilation would obtain an obvious promotion when the observations of the three-dimensional temperature and salinity measurements are applied, despite the sparse distribution [14-20]. According to the previous studies, the Argo T-S observations are assimilated and compared with the model state variables directly. Considering the continuous of the ocean dynamic evolution in space, the structure of the adjacent surroundings can be extrapolated to some degree. In order to improve the impact of Argo profile on the assimilation, an extending algorithm based on the Argo profile is developed and applied to present more reconstructed information. Then the impact of the reconstructed temperature profile (RTP) on the assimilation is evaluated. The paper is organized as follows: the data source and the details of the extending algorithm and the experiment configuration are introduced in Section 2; Section 3 refers to the result and discussion and focuses on the positive impact of the extending algorithm on the assimilation; at last, several final remarks are given in Section 4.

\section{Data and Methods}

\subsection{Data}

2.1.1. Argo Temperature and Salinity Profile. The Argo program is designed to observe large-scale subsurface ocean T/S profiles globally. The measurement starts near the surface and may reach $2000 \mathrm{~m}$ deep, with a complete cycle of 10 days [21]. In this study, the Argo profiles during Jan 2010 are selected and applied in the extending algorithm and the data assimilation. The source profiles are provided by the China Argo Real-Time Data Center (http://www.argo.org.cn/).

2.1.2. Sea Surface Temperature. In this study, AVHRR (Advanced Very High Resolution Radiometer) SST provided by NCDC/NOAA is employed. The product is merged by the satellite observations and large amounts of in situ SST data based on the optimal interpolation method; the spatial and temporal resolution are $0.25^{\circ} \times 0.25^{\circ}$ and 1 day, respectively [22]. Considering the precise accuracy of AVHRR, it is applied to initialize the extending algorithm.
2.1.3. EN4 Objective Analyses Dataset. The EN4 gridded dataset is a global quality controlled ocean T-S monthly objective analyses product, which is supplied by Hadley Centre of the UK Met Office and covers the period 1900 to present. Data from all types of ocean profiling instruments that provide temperature and salinity information are ingested into the dataset [23]. The spatial resolution is $1^{\circ} \times 1^{\circ}$ in the horizontal and 42 layers in the vertical. According to its high quality, the EN4 is employed as the reference to evaluate the impact of the RTP in the assimilation.

2.2. Extending Algorithm of Argo Temperature Profile. The Argo observations present an overview of the structure characteristic in the upper $2000 \mathrm{~m}$ ocean. Considering the discrete measurements of Argo, a piecewise fitting method is applied to obtain the continuous vertical temperature variation of each profile, accompanied by the vertical temperature gradient. According to Riser et al. [13], every single profile could be regarded as a representative in the adjacent region. Thus, the extending algorithm of Argo profile is developed to reconstruct the temperature structure of the neighbors. The position of reconstruction is determined by the grids of AVHRR surrounding each Argo profile. Since the vertical temperature gradient of the RTP could be approximated by the in situ Argo profile, the process of the extending algorithm is accomplished downward from the surface and initialized by the AVHRR SST.

Normally, the oceanic temperature has an obvious stratification structure in the vertical [24], including the Mixed Layer (ML), thermocline, and deep layers. As shown in Figure 1, the measurements of the Argo profile are divided into two parts by the Mixed Layer Depth (MLD): in the ML and below the ML. Considering the irregular fluctuation in the ML, simple curve fitting method is not applicable and hence the piecewise linear fitting strategy is employed. Thanks to the higher density of measurements, the temperature estimation between two observations is applicable and the error introduced by the piece linear fitting method is limited. Below the ML, the variation of temperature is likely to be consistent with an exponential function, so it is reasonable that the Gaussian fitting method is applied to obtain the complete temperature function $T\left(x_{A}, y_{A}, h\right)$. Since the vertical temperature function of depth is fitted, the corresponding vertical temperature gradient could be calculated easily.

In the following, some details of the fitting process and the calculation of vertical temperature gradient are illustrated. The classical $0.5^{\circ} \mathrm{C}$ threshold value from Monterey and Levitus [25] is chosen as the temperature criterion to determine the MLD of each Argo temperature profile. Let $h_{m}$ represent the MLD determined by the Argo profile, and then two groups of measure points could be obtained. Here we use Groups 1 and 2 denoting the measurements in the ML and below the ML, and then the fitting strategy is presented, respectively.

Group 1. For measure points in the ML, the depth satisfies $h \leq h_{m}$ and the piecewise linear function is employed. Suppose $\left(h_{i}, T\left(x_{A}, y_{A}, h_{i}\right)\right)$ and $\left(h_{i+1}, T\left(x_{A}, y_{A}, h_{i+1}\right)\right)$ stand 

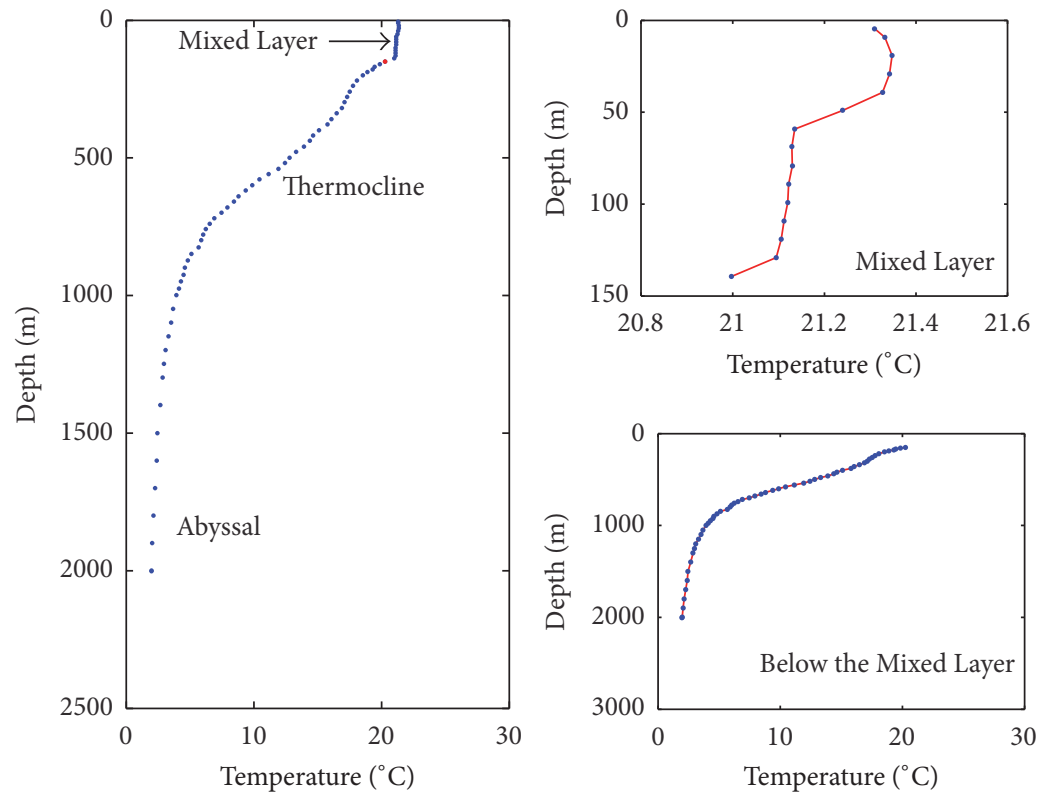

FIGURE 1: Strategy to divide the Argo measure points: the red dot represents the estimation of the MLD.

for arbitrary two adjacent measure points in the ML, where $x_{A}, y_{A}$ are the longitude and latitude of Argo profile and $h$ is the depth of measurements; the fitting function $T\left(x_{A}, y_{A}, h\right)$ between the two points is

$$
\begin{array}{r}
T\left(x_{A}, y_{A}, h\right)=T\left(x_{A}, y_{A}, h_{i}\right)+G_{i}\left(x_{A}, y_{A}\right) \cdot\left(h-h_{i}\right), \\
\left(h_{i} \leq h \leq h_{i+1} \leq h_{m}\right),
\end{array}
$$

where $G_{i}\left(x_{A}, y_{A}\right)$ represents the vertical gradient between $\left(h_{i}, T\left(x_{A}, y_{A}, h_{i}\right)\right)$ and $\left(h_{i+1}, T\left(x_{A}, y_{A}, h_{i+1}\right)\right)$, which could be calculated by

$$
\begin{aligned}
T^{\prime}\left(x_{A}, y_{A}, h\right) & =G_{i}\left(x_{A}, y_{A}\right) \\
& =\frac{T\left(x_{A}, y_{A}, h_{i+1}\right)-T\left(x_{A}, y_{A}, h_{i}\right)}{h_{i+1}-h_{i}} .
\end{aligned}
$$

Group 2. If the depth of the Argo measurements is beyond the MLD $h_{m}$, the Gaussian fitting procedure would be applied. Generally, the Gaussian fitting function has the form $\sum_{i=1}^{N} a_{i} \exp \left(-\left(\left(x-b_{i}\right) / c_{i}\right)^{2}\right)$; here $a_{i}, b_{i}$, and $c_{i}$ are the parameters of Gaussian function, which are determined by the least squares method [26]. $N$ represents the Gaussian order. Therefore the fitting temperature function below the ML could be written as

$$
\begin{array}{r}
T\left(x_{A}, y_{A}, h\right)=\sum_{i=1}^{N} a_{x_{A}, y_{A}}^{i} \exp \left(-\left(\frac{\left(h-b_{x_{A}, y_{A}}^{i}\right)}{c_{x_{A}, y_{A}}^{i}}\right)^{2}\right), \\
\left(h>h_{m}\right) .
\end{array}
$$

It is worth mentioning that these parameters of different profiles are not the same. Now the function $T\left(x_{A}, y_{A}, h\right)$ is continuously differentiable when $h>h_{m}$ and the derivative could be achieved by

$$
\begin{aligned}
& T^{\prime}\left(x_{A}, y_{A}, h\right)=-2 \sum_{i} \frac{a_{x_{A}, y_{A}}^{i}}{c_{x_{A}, y_{A}}^{i}}{ }^{2} \\
& \quad \times\left(h-b_{x_{A}, y_{A}}^{i}\right) \exp \left(-\left(\frac{\left(h-b_{x_{A}, y_{A}}^{i}\right)}{c_{x_{A}, y_{A}}^{i}}\right)^{2}\right) .
\end{aligned}
$$

The Argo profile starts from few meters deep normally. Let $h_{\min }$ represent the minimum depth of Argo profile, so if $h \leq h_{\text {min }}$ there are no measurements; here a constant function is applied to fit the temperature

$$
T\left(x_{A}, y_{A}, h\right)=T^{\mathcal{S}}\left(x_{A}, y_{A}\right)
$$

here $T^{\mathcal{S}}\left(x_{A}, y_{A}\right)$ stands for SST and the vertical temperature gradient is

$$
T^{\prime}\left(x_{A}, y_{A}, h\right)=0
$$

Since the gradient of each Argo profile at arbitrary depth $T^{\prime}\left(x_{A}, y_{A}, h\right)$ is available, we can obtain the approximation of vertical temperature gradient at the surrounded points $\left(x_{A 1}, y_{A 1}\right),\left(x_{A 2}, y_{A 2}\right),\left(x_{A 3}, y_{A 3}\right)$, and $\left(x_{A 4}, y_{A 4}\right)$. Because the distance between the Argo profile and the RTP is small, the variation of vertical temperature gradient between Argo profile and RTP could be ignored. Therefore in this study, the vertical temperature gradient of RTP $T^{\prime}\left(x_{\mathrm{RTP}}, y_{\mathrm{RTP}}, h\right)$ is taken equal to the value of Argo profile $T^{\prime}\left(x_{A}, y_{A}, h\right)$ approximately; here $\left(x_{\mathrm{RTP}}, y_{\mathrm{RTP}}\right)$ is the longitude and latitude 


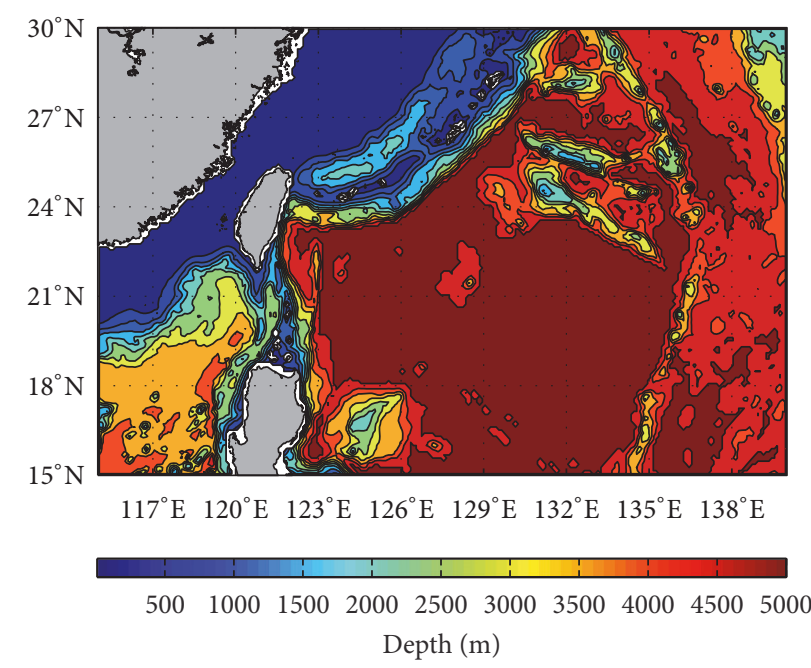

FIGURE 2: Model domain and bathymetry (m).

of the RTP. Initialized by the AVHRR SST, the reconstruction of the RTP can be conducted by the equation

$$
\begin{aligned}
T\left(x_{\mathrm{RTP}}, y_{\mathrm{RTP}}, h_{i+1}\right)= & T\left(x_{\mathrm{RTP}}, y_{\mathrm{RTP}}, h_{i}\right) \\
& +T^{\prime}\left(x_{\mathrm{RTP}}, y_{\mathrm{RTP}}, h_{i}\right) \\
& \cdot\left(h_{i+1}-h_{i}\right),
\end{aligned}
$$

where $h_{i}\left(i=0,1, \ldots, N_{R P}\right)$ is the reconstruction depth of RTP. Finally, four more temperature profiles could be reconstructed by the extending algorithm for each Argo profile.

2.3. Model and Assimilation Configuration. In order to evaluate the impact of the derived RTP on the assimilation, an experiment based on ROMS and 4DVAR is conducted. ROMS is a free-surface, hydrostatic, primitive equation model discretized with a terrain following vertical coordinate system [27]. The model domain is shown in Figure 2, which covers the area from $15^{\circ} \mathrm{N}$ to $30^{\circ} \mathrm{N}, 115^{\circ} \mathrm{E}$ to $140^{\circ} \mathrm{E}$, with an eddy-resolved horizontal resolution of $0.1^{\circ} \times 0.1^{\circ}$ (about $10 \mathrm{~km}$ ) and $30 \mathrm{~S}$-coordinate layers in the vertical. The level 2.5 Mellor Yamada [28] scheme is adopted as the parameterization of the vertical mixing process. The temperature and salinity field is initialized by the Levitus climatology dataset, while the free surface and velocity are set as 0 . The ETOPO5 data [29] is employed to produce the bathymetry field. The minimum and maximum depths in the whole domain are set to be 10 and $5000 \mathrm{~m}$, respectively. The wind field uses a daily mean wind field from the Cross-Calibrated Multi-Platform (CCMP) ocean surface wind product [30] with a horizontal resolution of $0.25^{\circ} \times 0.25^{\circ}$. The other daily atmospheric forcing fields, including heat fluxes, solar radiation fluxes, evaporation-precipitation (E-P), air temperature, and specific humidity, are obtained from the US National Centers of Environmental Prediction (NCEP) reanalysis [31] with a horizontal resolution of $1.875^{\circ} \times 1.875^{\circ}$. Finally the monthly
TABLE 1: Summary of assimilation data in cases 1-4.

\begin{tabular}{lcccc}
\hline & Case 1 & Case 2 & Case 3 & Case 4 \\
\hline Temperature & None & Argo & RTP + Argo & SST + Argo \\
Salinity & None & Argo & Argo & Argo \\
\hline
\end{tabular}

temperature, salinity, sea surface height, and velocity field from SODA provide the lateral boundary conditions.

After 5 years' integration starts from Jan 1, 2005, the forward model comes to an ocean dynamic balanced state and the outcome is employed for the calculation of background error covariance matrix. The choice of parameters for modeling the background error covariance matrix (D) and the observation error covariance matrix $(\mathbf{R})$ are designed as Moore et al. [32]. The background errors of all initial condition control variable components of $\mathbf{D}$ were $50 \mathrm{~km}$ in the horizontal and $30 \mathrm{~m}$ in the vertical. Horizontal correlation scales chosen for the background surface forcing error components of $\mathbf{D}$ were $300 \mathrm{~km}$ for wind stress and $100 \mathrm{~km}$ for heat and freshwater fluxes. The correlation lengths for the background open boundary condition error components of D were chosen to be $100 \mathrm{~km}$ in the horizontal and $30 \mathrm{~m}$ in the vertical. Observation errors were assumed to be uncorrelated in space and time, and the variances along the main diagonal of $\mathbf{R}$ were assigned as a combination of measurement error and the error of representativeness which in general are additive. Measurement errors of Argo T-S and AVHRR SST were chosen as the following standard deviations: $0.4^{\circ} \mathrm{C}$ for SST; $0.1^{\circ} \mathrm{C}$ and 0.01 for Argo T-S. Meanwhile, the standard deviation of RTP is roughly determined as $0.1^{\circ} \mathrm{C}$.

In order to evaluate the impact of RTP on the assimilation, an experiment including four cases is designed. Case 1 is conducted without assimilation and considered as the control experiment. The Argo temperature and salinity observations are assimilated in case 2, while the derived RTP and Argo profile are applied in case 3 . Because some information of AVHRR SST is introduced in the RTP reconstruction process, case 4 is designed in order to evaluate and remove the influence of SST, in which only the information from the top layer of RTP is assimilated. The details of the experiment are presented in Table 1.

\section{Result and Discussion}

Firstly, the Argo observations are selected and applied in the extending process and the assimilation experiment. In the extending algorithm, the reconstruction of RTP is conducted in the upper $1000 \mathrm{~m}$, with totally 25 levels in the vertical and 20 levels in the upper $500 \mathrm{~m}$. As the gradient of RTP is approximated by the nearby Argo, the profiles with maximum measure depth less than $1000 \mathrm{~m}$ are abandoned. Besides, the observations with abnormal measurements are removed. The RTP is reconstructed and assimilated in the experiment based on the extending algorithm. Finally, the accuracy analysis of the experiment is conducted with EN4, to evaluate the impact of the extending algorithm on the assimilation. 


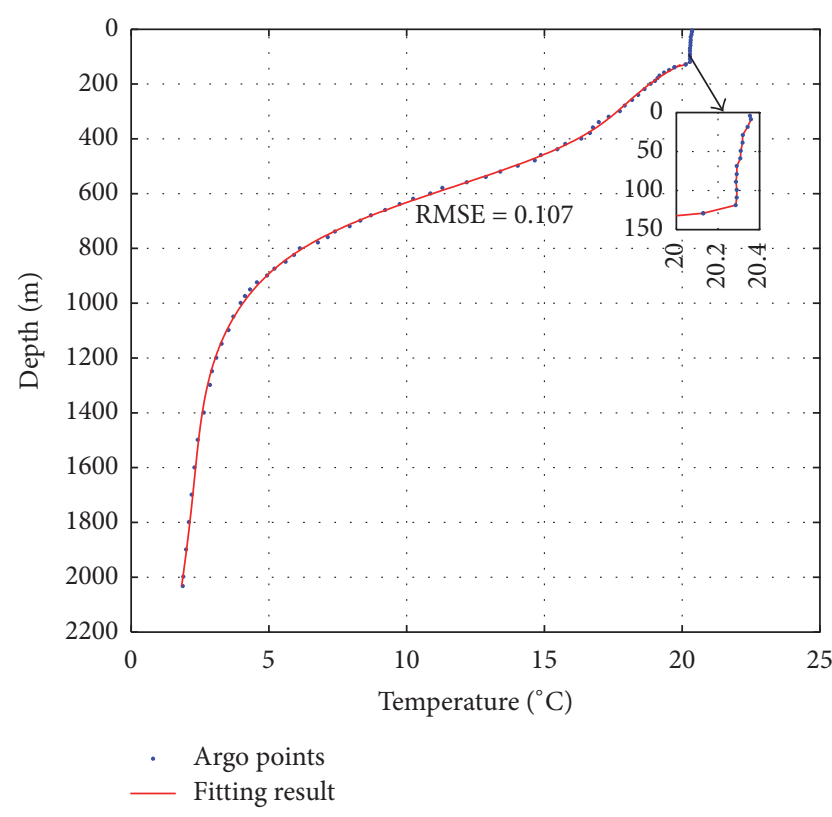

(a)

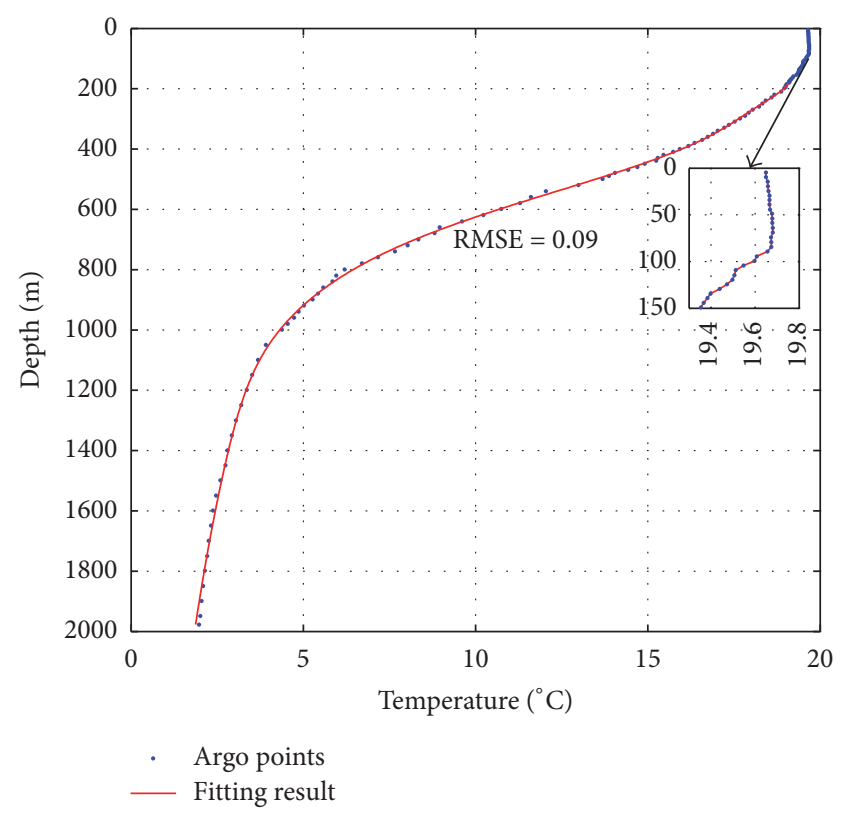

(b)

FIGURE 3: Fitting result of Argo profile, platform, and cycle number: 2900715_162 (a) and 2900683_110 (b).

3.1. Fitting Result of Argo Profile. For each Argo profile, the piecewise fitting method is applied to obtain a continuous temperature function of depth. In the ML, the piecewise linear function is employed to present the temperature variation in the vertical, while the Gaussian function fitting is used below the ML. The order of Gaussian function $N$ is determined by the number of measurements in Argo profile; here 20,50, and 90 are taken as the thresholds of Gaussian orders 2, 3, 4, and 5. Since the temperature function of Argo is obtained by the fitting procedure, the vertical temperature gradient could be calculated by (2), (4), and (6). Two fitting results are shown in Figure 3. We can see that the fitting result is highly consistent with the measurements of Argo and the Root Mean Squared Error (RMSE) being $0.107^{\circ} \mathrm{C}$ and $0.09^{\circ} \mathrm{C}$, respectively. As the reconstruction of the RTP is realized downward based on the vertical temperature gradient, the good performance of the fitting process would benefit the reconstruction a lot.

3.2. Temperature Reconstruction. As mentioned above, four grids of AVHRR surrounding each Argo profile are identified as the position of RTP and denoted as RTP 1, RTP 2, RTP 3, and RTP 4. The distribution of the Argo profiles and RTPs are presented in Figure 4; four times more reconstructed profiles could be obtained by the benefit of the proposed extending algorithm of Argo temperature profile. In Section 3.1, the temperature gradient functions of depth at the Argo measure position are obtained, and then the vertical temperature gradient of RTP is approximated by corresponding Argo profile. Finally, by the combination of AVHRR SST and the derived vertical temperature gradient, the reconstruction of RTP could be realized as (7).

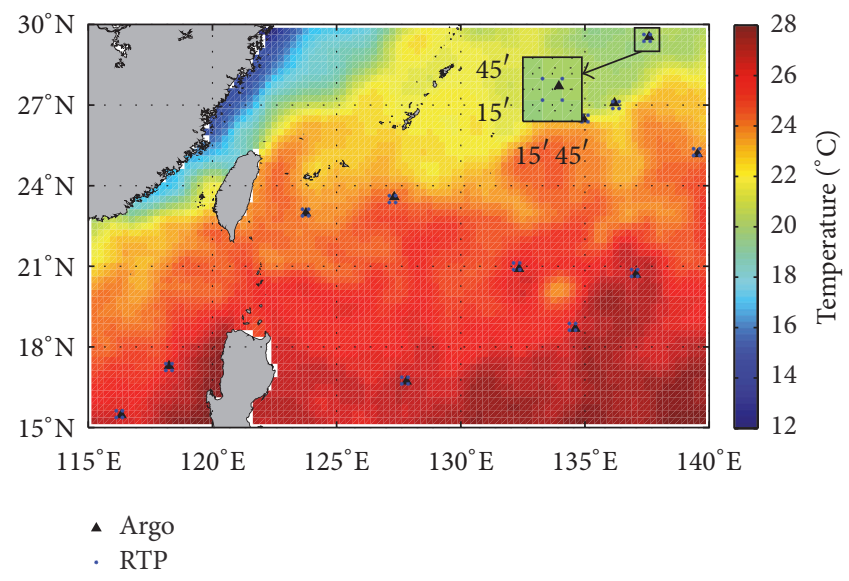

FIGURE 4: Distribution of Argo and RTP in the reconstruction (Jan 06, 2010): the background represents SST $\left({ }^{\circ} \mathrm{C}\right)$.

In the reconstruction of RTP, the four profiles surrounding each Argo are reconstructed by the same temperature gradient, which leads to the similar shape of temperature profiles. As shown in Figure 5, the profile reconstruction is conducted above $1000 \mathrm{~m}$. Though the temperature variation of RTP is similar with the Argo profile, some different characteristics are introduced by the initial value, such as the thermocline. Because SST is a presentation of complex airsea interaction process, the difference between the initial conditions of different RTP is distinct. Since the reconstruction of RTP is accomplished, the extending temperature profiles could be applied to enhance the assimilation. 


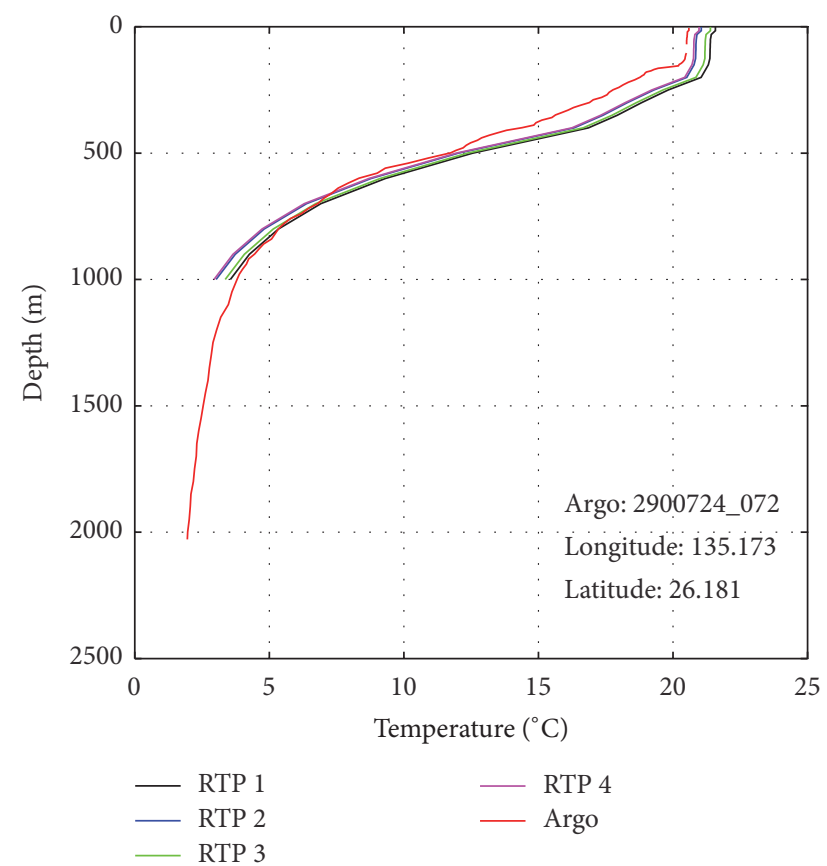

(a)

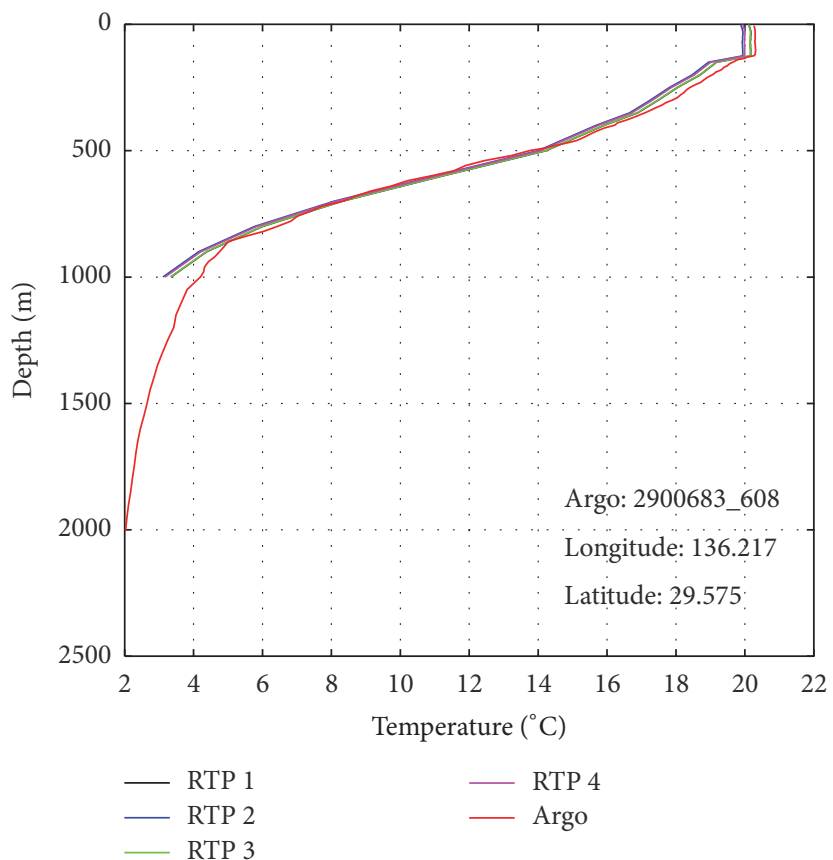

(b)

FIGURE 5: Two samples of reconstructed RTP and the corresponding Argo temperature profile.

3.3. Analysis of the Assimilation Results. Based on the RTP derived by the extending algorithm of Argo temperature profile, more temperature information of the surroundings is obtained and would benefit the T-S assimilation with more "observations." To validate this, an experiment focusing on the T-S assimilation is conducted based on the ROMS and 4DVAR. Respectively, the Argo profile and RTP are assimilated, and then the results are evaluated by EN4. Because only the temperature profile is reconstructed by the developed algorithm, the evaluation mainly focuses on the enhancement of temperature simulation.

Firstly, the monthly mean of the assimilation results is calculated and interpolated to grid of the EN4. In the comparison, the Horizontal Absolute Bias (HAB) and Vertical Absolute Bias (VAB) above $5000 \mathrm{~m}$ are considered. The calculation formula is

$$
\begin{aligned}
& \mathrm{HAB}=\frac{1}{N_{1}} \sum_{k=1}^{N_{1}}|M(i, j, k)-E(i, j, k)|, \\
& \mathrm{VAB}=\frac{1}{N_{2} N_{3}} \sum_{j=1}^{N_{2}} \sum_{i=1}^{N_{3}}|M(i, j, k)-E(i, j, k)|,
\end{aligned}
$$

where $M$ and $E$ represent the model assimilation result and EN4 data, $N_{1}$ is the vertical layers above $5000 \mathrm{~m}$, and $N_{2}$ and $\mathrm{N}_{3}$ represent the horizontal dimensions of EN4.

As shown in Figure 6, the accuracy of the three assimilation cases is all promoted, both in the horizontal and vertical, which is benefit from the assimilated temperature and salinity observations. Figure 6(a) presents the HAB of the four cases in the experiment; it is obvious that the accuracy at the northeastern region of Taiwan is largely enhanced when the T-S observations are assimilated, both in cases 2-4. Compared with case 2, the addition of the RTP in case 3 shows positive impact on the assimilation, and the simulation of the ocean temperature near the Kuroshio $\left(120^{\circ} \mathrm{E} \sim 135^{\circ} \mathrm{E}, 25^{\circ} \mathrm{N} \sim 30^{\circ} \mathrm{N}\right)$ is improved distinctly. From the VAB comparison shown in Figure 6(b), we can see the benefit of T-S assimilation can reach as deep as $2500 \mathrm{~m}$, which is consistent with the measure depth of Argo. In the comparison between cases 2 and 3, the enhancement mainly occurs at the upper $500 \mathrm{~m}$, not the reconstruction depth $1000 \mathrm{~m}$; here the unbalanced distribution of reconstruction depth may be blamed. Moreover, the VAB curve of case 4 is almost the same as case 2 , which indicates that no more obvious enhancements are obtained by the addition of SST; considering that only few SST is assimilated in case 4, the small enhancement is reasonable.

In the following, the analysis focusing on the temperature section of the experiment is conducted to confirm the impact of RTP on the assimilation. The differences between cases 1-4 and EN4 are illustrated in Figure 7, including the zonally and meridionally averaged sections. From Figure 7(a), we can see the simulation in the southern $20^{\circ} \mathrm{N}$ is better than the north; the temperature of the upper layers is smaller than EN4, which is in contrast with the deeper layers. Because the Luzon Strait along $20^{\circ} \mathrm{N}$ is the channel of water exchange between South China Sea and the Pacific Ocean and the origin of the Kuroshio, more difficulty is brought into the simulation by the anfractuous dynamic behavior. For the same reason, similar characteristics of meridionally averaged difference are presented in Figure 7(b).

After that, the absolute difference is averaged zonally and meridionally from top to bottom. As the results shown 


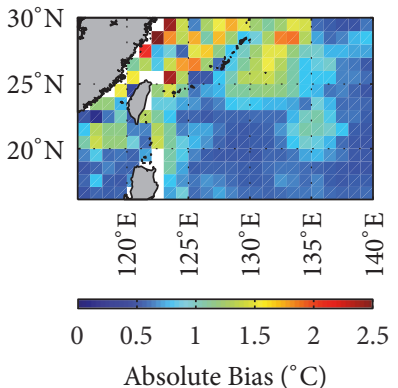

(a1)

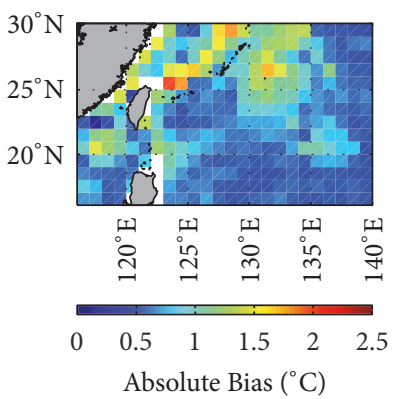

(a3)

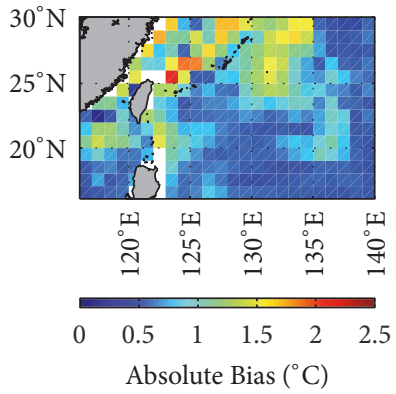

(a2)

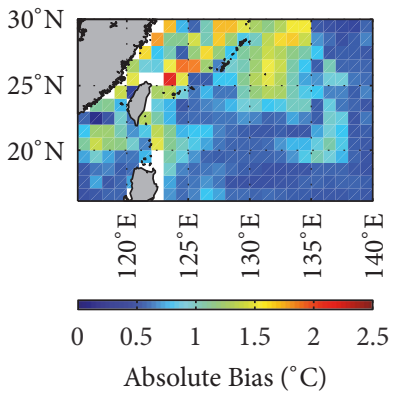

(a4)

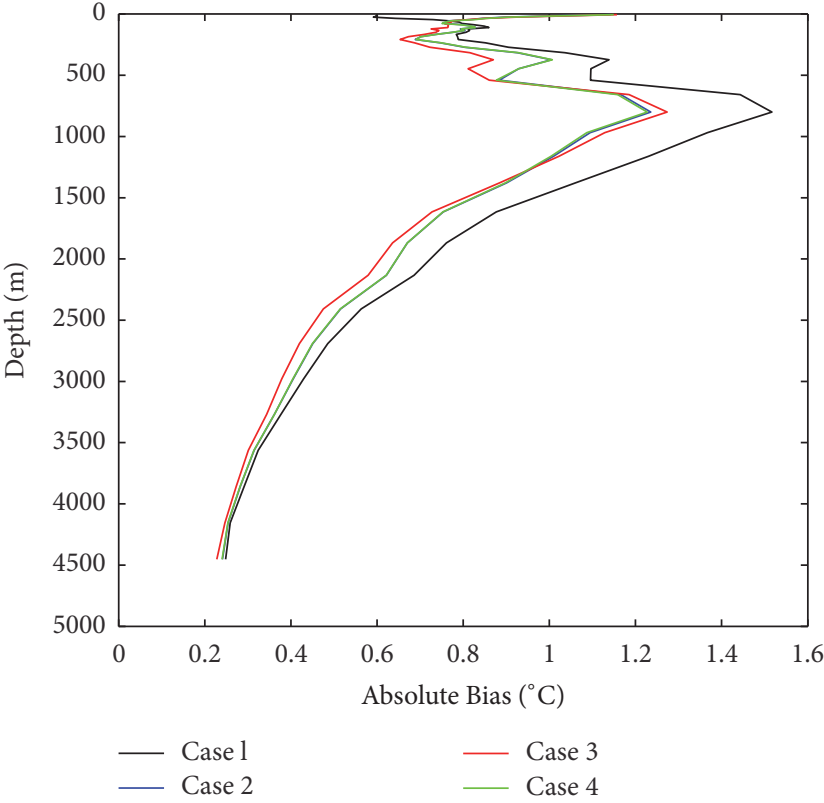

(b)

FIgURE 6: HAB (a) and VAB (b) of experiment result compared with EN4: symbols (a1)-(a4) are the HAB of cases 1-4, respectively.

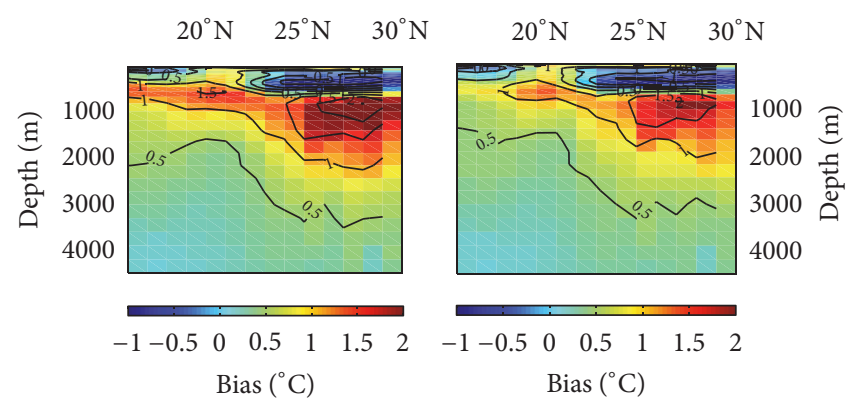

(a1)
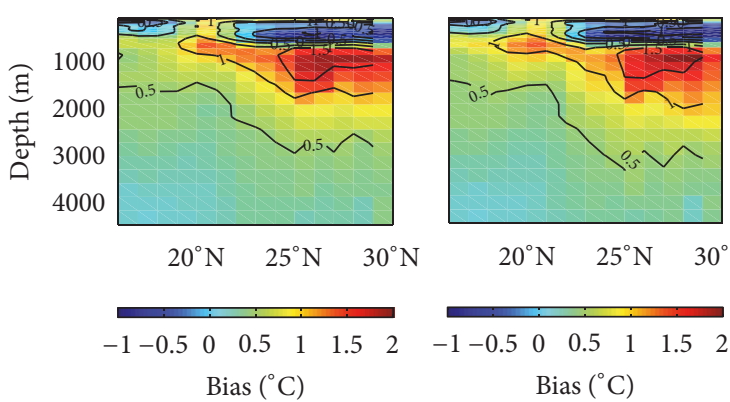

(a3)

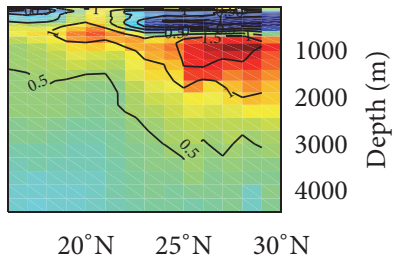

(a4)

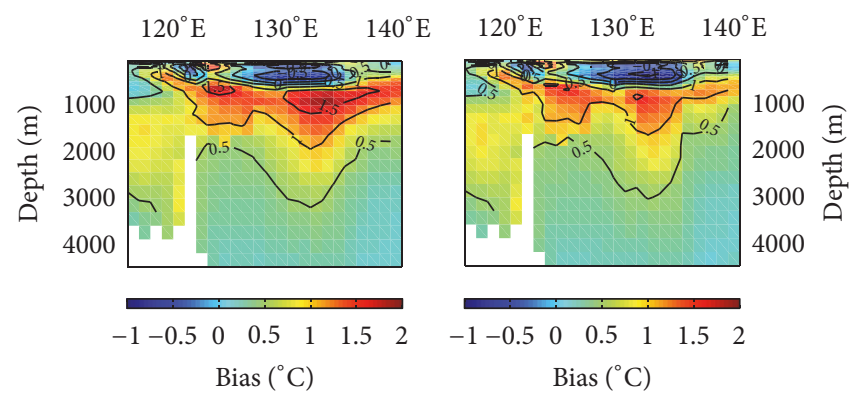

(b1)
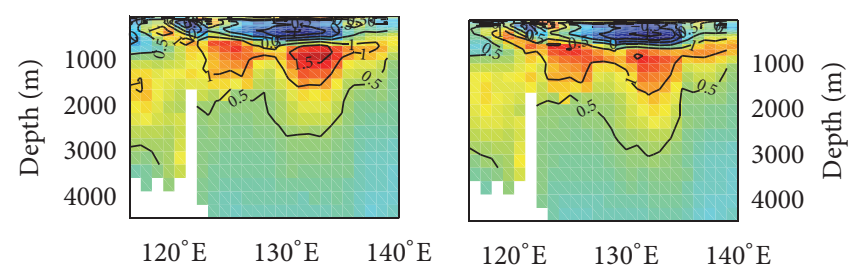

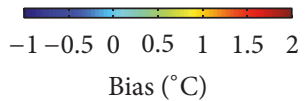

(b3) (b2)

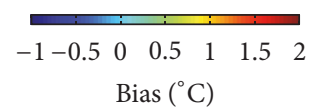

(b4)

(a)

(b)

FIGURE 7: Zonally (a1-a4) and meridionally (b1-b4) averaged section of the difference between EN4 and the four cases. 


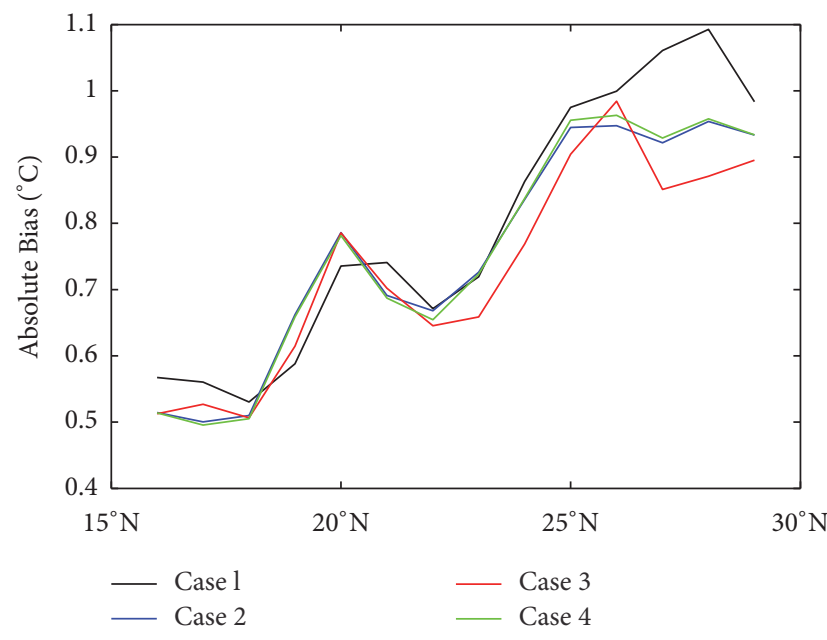

(a)

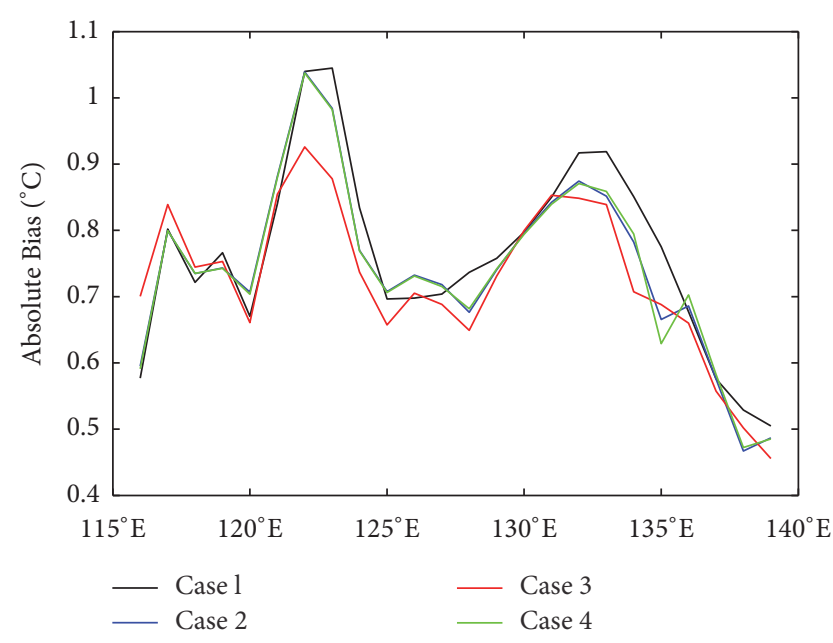

(b)

FIGURE 8: Mean difference averaged zonally (a) and meridionally (b) over the whole depth of cases 1-4: here the absolute difference is applied.

TABLE 2: Error statistics of the experiment.

\begin{tabular}{lcccc}
\hline & Case 1 & Case 2 & Case 3 & Case 4 \\
\hline A-Bias $\left({ }^{\circ} \mathrm{C}\right)$ & 0.792 & 0.7568 & 0.7305 & 0.7568 \\
RMSE $\left({ }^{\circ} \mathrm{C}\right)$ & 1.0065 & 0.9610 & 0.9311 & 0.9621 \\
\hline
\end{tabular}

in Figure 8, the influence of Kuroshio on the simulation is represented clearly. Moreover, case 3 added that the RTP achieves the best accuracy, and the positive impact of the proposed extending algorithm on the assimilation is well validated.

Table 2 presents the error statistics of the experiment, including the global average of Absolute Bias (A-Bias) and RMSE. It can be seen that the most accurate result is provided by case 3, whose A-Bias and RMSE are $0.7305^{\circ} \mathrm{C}$ and $0.9311^{\circ} \mathrm{C}$, respectively. Cases 2 and 4 are the medium ones and almost achieve the same accuracy. Therefore the contribution of SST observations introduced in the reconstruction algorithm could be ignored. Compared with the model simulation results, the A-Bias between cases 2 and 4 and EN4 are both reduced by $4.4 \%$, while case 3 achieves a proportion of $7.8 \%$. The benefits of Argo T-S observations and RTP are presented clearly, $4.4 \%$ enhancement for the Argo T-S observations and $3.4 \%$ for the RTP. Moreover, the similar analysis of RMSE has the same conclusion, and the benefit of the RTP is comparable with Argo T-S observations in the assimilation.

\section{Conclusion}

Actually, data assimilation could be considered as an error adjustment procedure to enhance the ocean modeling, by minimizing the difference between model and observations, so sufficient and accurate observations are demanded in the process. Considering the sparse distribution of Argo T-S measurements, an extending algorithm is developed to reconstruct the temperature profile at the AVHRR SST points surrounding each Argo. Therefore four times more reconstructed temperature profiles (RTPs) are obtained. Then an experiment including four cases is designed to evaluate the impact of RTP on the assimilation and the validation is conducted by the comparison with the EN4 dataset. The analysis indicates that the accuracy of cases 2 and 3 with Argo and RTP assimilated both obtain a promotion in the horizontal and vertical. Compared with the control experiment, the assimilation of the Argo profiles achieves an enhancement of $4.4 \%$ and $7.8 \%$ for case 3 . Because the influence of SST introduced in the reconstruction process can be ignored by the comparison of cases 2 and 4, the net enhancement of RTP introduced in the assimilation is $3.4 \%$, which is comparable with the impact of Argo T-S observations. Generally speaking, the RTP generated by the extending algorithm in the paper does have a positive impact on the assimilation of ocean modeling.

\section{Conflicts of Interest}

The authors declare that there are no conflicts of interest regarding the publication of this paper.

\section{Acknowledgments}

The study is supported by the National Key Research and Development Program of China under Contract no. 2016YFC1401800; the National Natural Science Foundation of China under Contract no. 41576176; the Key Project of Science and Technology of Weihai under Contract no. 2014DXG J14; and the Disciplinary Construction Guide Foundation of Harbin Institute of Technology at Weihai under Contract no. WH20140206.

\section{References}

[1] T. Ezer and G. L. Mellor, "Continuous assimilation of Geosat altimeter data into a three- dimensional primitive equation Gulf 
Stream model," Journal of Physical Oceanography, vol. 24, no. 4, pp. 832-847, 1994.

[2] D. B. Haidvogel, H. G. Arango, K. Hedstrom, A. Beckmann, P. Malanotte-Rizzoli, and A. F. Shchepetkin, "Model evaluation experiments in the North Atlantic Basin: Simulations in nonlinear terrain-following coordinates," Dynamics of Atmospheres and Oceans, vol. 32, no. 3-4, pp. 239-281, 2000.

[3] E. Di Lorenzo, "Seasonal dynamics of the surface circulation in the Southern California Current System," Deep-Sea Research Part II: Topical Studies in Oceanography, vol. 50, no. 14-16, pp. 2371-2388, 2003.

[4] M. S. Dinniman, J. M. Klinck, and W. O. Smith Jr., "Crossshelf exchange in a model of the Ross Sea circulation and biogeochemistry," Deep-Sea Research Part II: Topical Studies in Oceanography, vol. 50, no. 22-26, pp. 3103-3120, 2003.

[5] P. Marchesiello, J. C. McWilliams, and A. Shchepetkin, "Equilibrium structure and dynamics of the California current system," Journal of Physical Oceanography, vol. 33, no. 4, pp. 753-783, 2003.

[6] C. Dong, E. Y. Idica, and J. C. McWilliams, "Circulation and multiple-scale variability in the Southern California Bight," Progress in Oceanography, vol. 82, no. 3, pp. 168-190, 2009.

[7] S. Zhang and A. Rosati, "An inflated ensemble filter for ocean data assimilation with a biased coupled GCM," Monthly Weather Review, vol. 138, no. 10, pp. 3905-3931, 2010.

[8] H. E. Hurlburt, "Dynamic transfer of simulated altimeter data into subsurface information by a numerical ocean model.," Journal of Geophysical Research: Atmospheres, vol. 91, no. 2, pp. 2372-2400, 1986.

[9] M. Cooper and K. Haines, "Altimetric assimilation with water property conservation," Journal of Geophysical Research: Oceans, vol. 101, no. 1, pp. 1059-1077, 1996.

[10] D. N. Fox, W. J. Teague, C. N. Barron et al., "The Modular Ocean Data Assimilation System (MODAS)," Journal of Atmospheric \& Oceanic Technology, vol. 15, no. 1, pp. 22-28, 2002.

[11] C. Yan, J. Zhu, R. Li, and G. Zhou, "Roles of vertical correlations of background error and T-S relations in estimation of temperature and salinity profiles from sea surface dynamic height," Journal of Geophysical Research: Oceans, vol. 109, no. 8, pp. C08010-18, 2004.

[12] S. Ratheesh, R. Sharma, and S. Basu, "Projection-Based Assimilation of Satellite-Derived Surface Data in an Indian Ocean Circulation Model," Marine Geodesy, vol. 35, no. 2, pp. 175-187, 2012.

[13] S. C. Riser, H. J. Freeland, D. Roemmich et al., "Fifteen years of ocean observations with the global Argo array," Nature Climate Change, vol. 6, no. 2, pp. 145-153, 2016.

[14] M. Kamachi, T. Kuragano, H. Ichikawa et al., "Operational data assimilation system for the Kuroshio south of Japan: Reanalysis and validation," Journal of Oceanography, vol. 60, no. 2, pp. 303312, 2004

[15] P. R. Oke, A. Schiller, D. A. Griffin, and G. B. Brassington, "Ensemble data assimilation for an eddy-resolving ocean model of the Australian region," Quarterly Journal of the Royal Meteorological Society, vol. 131, no. 613, pp. 3301-3311, 2006.

[16] J. A. Cummings, "Operational multivariate ocean data assimilation," Quarterly Journal of the Royal Meteorological Society, vol. 131, no. 613, pp. 3583-3604, 2006.

[17] M. J. Martin, A. Hines, and M. J. Bell, "Data assimilation in the FOAM operational short-range ocean forecasting system: A description of the scheme and its impact," Quarterly Journal of the Royal Meteorological Society, vol. 133, no. 625 B, pp. 981-995, 2007.

[18] P. R. Oke, G. B. Brassington, D. A. Griffin, and A. Schiller, "The Bluelink ocean data assimilation system (BODAS)," Ocean Modelling, vol. 21, no. 1-2, pp. 46-70, 2008.

[19] B. Huang, Y. Xue, and D. W. Behringer, "Impacts of Argo salinity in NCEP global ocean data assimilation system: the tropical Indian Ocean," Journal of Geophysical Research: Oceans, vol. 113, no. 8, Article ID C08002, 2008.

[20] G. C. Smith and K. Haines, "Evaluation of the S(T) assimilation method with the Argo dataset," Quarterly Journal of the Royal Meteorological Society, vol. 135, no. 640, pp. 739-756, 2009.

[21] D. Roemmich and J. Gilson, "The 2004-2008 mean and annual cycle of temperature, salinity, and steric height in the global ocean from the Argo Program," Progress in Oceanography, vol. 82, no. 2, pp. 81-100, 2009.

[22] R. W. Reynolds, T. M. Smith, C. Liu, D. B. Chelton, K. S. Casey, and M. G. Schlax, "Daily high-resolution-blended analyses for sea surface temperature," Journal of Climate, vol. 20, no. 22, pp. 5473-5496, 2007.

[23] S. A. Good, M. J. Martin, and N. A. Rayner, "EN4: Quality controlled ocean temperature and salinity profiles and monthly objective analyses with uncertainty estimates," Journal of Geophysical Research: Oceans, vol. 118, no. 12, pp. 6704-6716, 2013.

[24] B. Cushman-Roisin and J. Beckers, "Equations Governing Geophysical Flows," in Introduction to Geophysical Fluid Dynamics - Physical and Numerical Aspects, vol. 101 of International Geophysics, pp. 99-129, Elsevier, 2011.

[25] G. Monterey and S. Levitus, Seasonal Variability of Mixed Layer Depth for the World Ocean, U S Gov Printing Office, Washington, DC, USA, 1997.

[26] C. L. Lawson and R. J. Hanson, Solving Least Squares Problems, Prentice-Hall, Englewood Cliffs, NJ, USA, 1974.

[27] A. F. Shchepetkin and J. C. McWilliams, "The regional oceanic modeling system (ROMS): a split-explicit, free-surface, topography-following-coordinate oceanic model," Ocean Modelling, vol. 9, no. 4, pp. 347-404, 2005.

[28] G. L. Mellor and T. Yamada, "Development of a turbulence closure model for geophysical fluid problems," Reviews of Geophysics \& Space Physics, vol. 20, no. 4, pp. 851-875, 1982.

[29] M. Edwards, "Data announcement 88-MGG-02: Digital relief of the surface of the earth," National Oceanic and Atmospheric Administration, 1988.

[30] R. Atlas, R. N. Hoffman, J. Ardizzone et al., "A cross-calibrated, multiplatform ocean surface wind velocity product for meteorological and oceanographic applications," Bulletin of the American Meteorological Society, vol. 92, no. 2, pp. 157-174, 2011.

[31] E. Kalnay, M. Kanamitsu, R. Kistler et al., "The NCEP/NCAR 40-year reanalysis project," Bulletin of the American Meteorological Society, vol. 77, no. 3, pp. 437-471, 1996.

[32] A. M. Moore, H. G. Arango, G. Broquet et al., "The Regional Ocean Modeling System (ROMS) 4-dimensional variational data assimilation systems. Part II - Performance and application to the California Current System.", Progress in Oceanography, vol. 91, no. 1, pp. 50-73, 2011. 

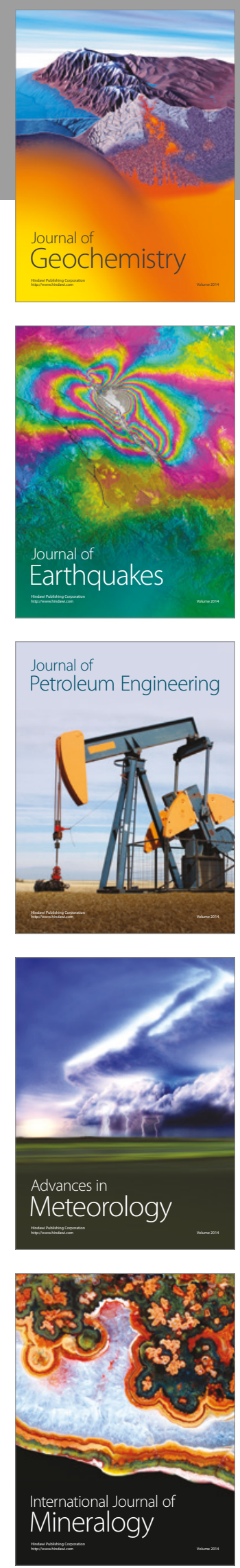
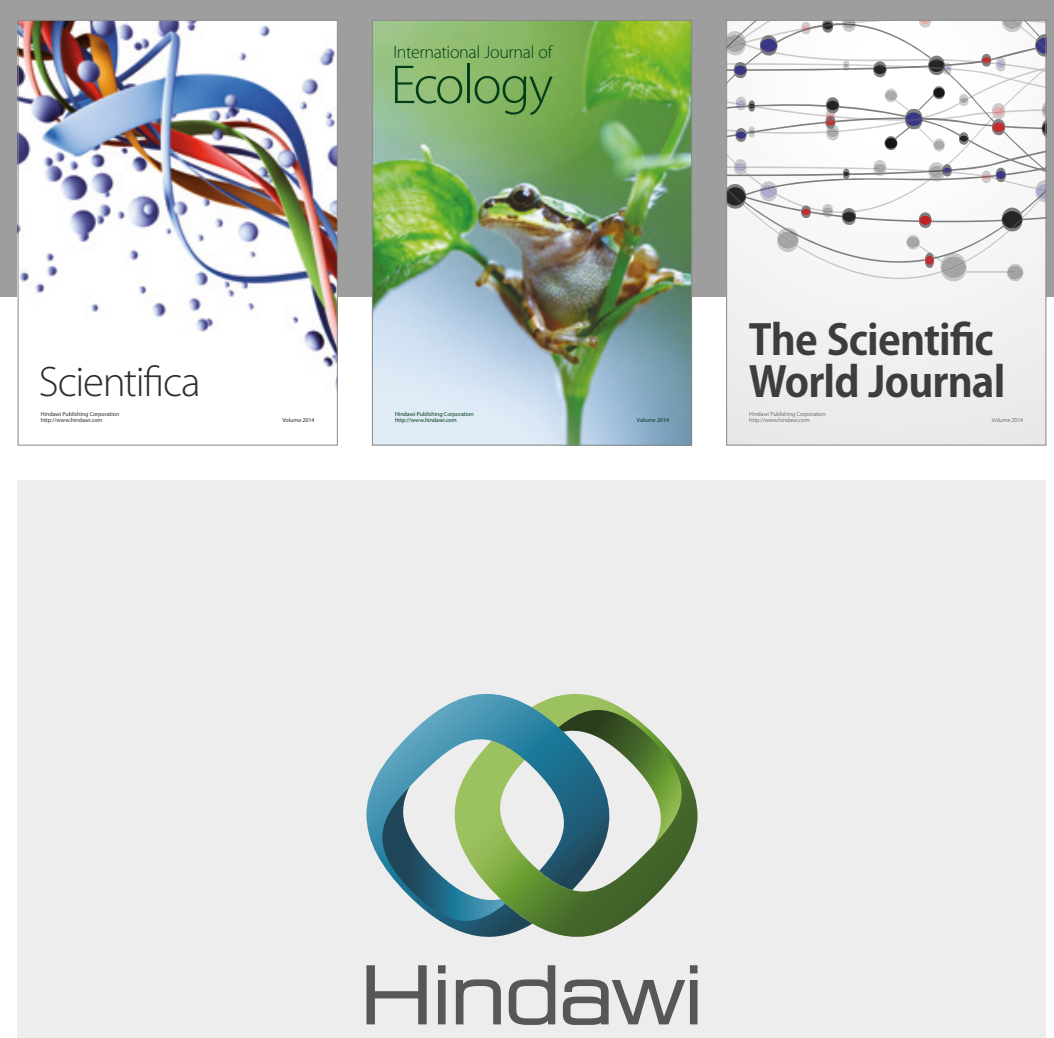

Submit your manuscripts at

https://www.hindawi.com
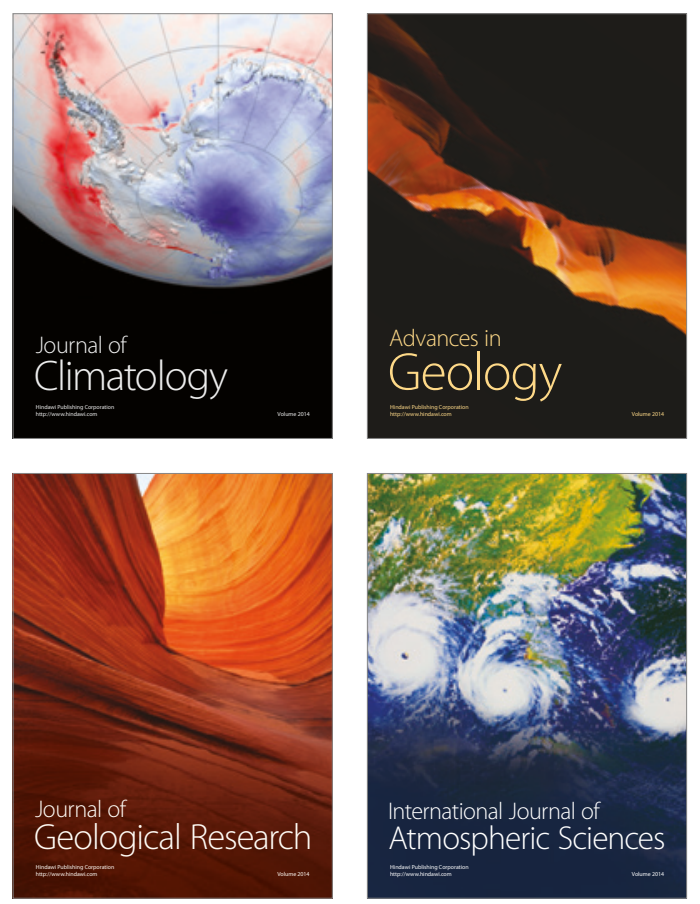

The Scientific

World Journal
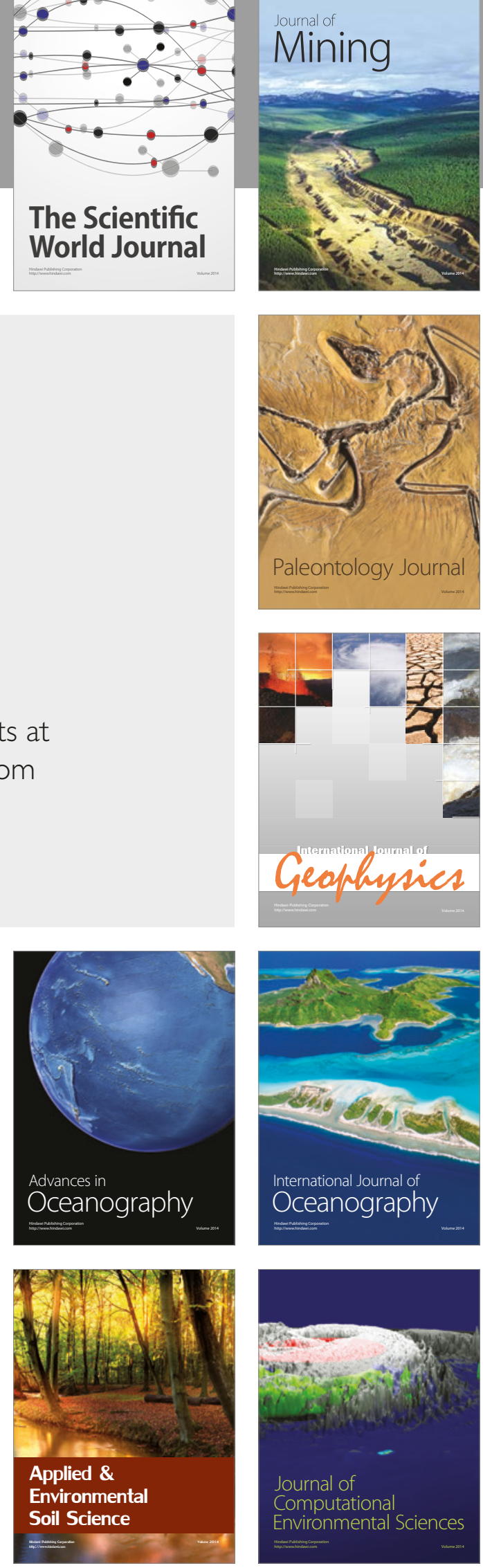